\title{
Association between folate intake from different food sources in Norway and homocysteine status in a dietary intervention among young male adults
}

\author{
Tonje Holte Stea ${ }^{1}$, Solveig Uglem ${ }^{2}$, Margareta Wandel ${ }^{2}$, Mohammad Azam Mansoor ${ }^{3}$ and Wenche Frølich ${ }^{1}$ \\ ${ }^{1}$ Norwegian School of Hotel Management, University of Stavanger, N-4036 Stavanger, Norway \\ ${ }^{2}$ Department of Nutrition, Institute for Basic Medical Sciences, University of Oslo, N-0316 Oslo, Norway \\ ${ }^{3}$ Department of Natural Sciences, Agder University, N-4604 Kristiansand, Norway
}

(Received 13 October 2008 - Revised 4 February 2009 - Accepted 5 February 2009 - First published online 31 March 2009)

The aim of the present investigation was to study the effect of a dietary intervention which combined nutrition information with increased availability of vegetables, fruits and wholegrain bread. The effect of the intervention was determined by changes in the intake of vegetables, fruits, wholegrain bread and estimated nutrients. Furthermore, the study investigated whether changes in relative contribution from different food sources of folate were related to changes in the concentration of plasma total homocysteine (p-tHcy). The 5-month intervention study included 376 male recruits from the Norwegian National Guard, Vaernes (intervention group) and 105 male recruits from the Norwegian National Guard, Heggelia (control group). The study resulted in an increase in the total consumption of vegetables, fruits, berries and juice $(P<0 \cdot 001)$ and of wholegrain bread $(P<0.001)$. The participants in the intervention group showed a higher increase in the intake of dietary fibre $(P<0.001)$ and folate $(P<0.001)$ compared with the control group. The relative contribution of folate intake from fruits, vegetables and wholegrain bread was higher in the intervention group compared with the control group $(P<0.001$ for all). The increased intake of folate from wholegrain bread was inversely associated with a reduced concentration of p-tHcy $(P=0.017)$. In summary, the dietary intervention resulted in an increased intake of vegetables, fruits and wholegrain bread and a subsequent increase in folate intake from these food components. Reduction in the concentration of p-tHcy was significantly related to an increased folate intake due to an increased consumption of wholegrain bread.

Dietary intervention studies: Homocysteine: Young male adults

Young male adults are an important but difficult target group to reach when promoting healthy diets. The period when young men are moving away from home for the first time, for example, when they are enrolled in military service, often involves changes in dietary habits ${ }^{(1)}$.

In several European countries, descriptive studies have shown that subjects in all age groups, including children and adolescents, have an increased intake of high-energy foods, lower intakes of vegetables, fruits and wholegrain products and lower level of physical activity ${ }^{(2-6)}$. Unhealthy dietary habits from childhood and adolescence (11-16 years of age) have been demonstrated to be carried over into adulthood and thereby affect the prevalence of cardiovascular risk factors $^{(7-9)}$. A follow-up study of young men and women (mean age 25 and 33 years, respectively) showed that their eating behaviour from childhood was a significant predictor for their intake of fruits, vegetables, wholegrain and sugar-rich foods ${ }^{(10)}$. These studies have confirmed the need to promote and improve healthy eating habits in early age and with special focus on individuals with low intakes of fruits, vegetables and wholegrain products.

A literature review suggested that improved eating habits can be achieved by a variety of approaches in different subgroups in a population $^{(11)}$. Dietary interventions among adults have often been focused on strategies to increase nutritional knowledge $^{(12,13)}$. A significant improvement in dietary habits has been shown to require a combination of different approaches when targeting specific population groups ${ }^{(14-16)}$. Intervention studies in the age group of 6-18 years have proved most effective when dietary information has been combined with increased availability of fruits and vegetables ${ }^{(17,18)}$. Despite positive effects from these studies mentioned, an intervention programme focusing on both nutritional information and increased availability of healthier foods among pupils in secondary schools showed no significant changes in school-based eating over a 2 -year period ${ }^{(19)}$. Data from various intervention programmes have demonstrated how difficult it is to achieve changes in dietary habits in children and adolescents.

Previously, adults have been the main target group in studies that have evaluated the effects of dietary interventions on changes in cardiovascular risk factors. An 8-month intervention study with a low-fat $(<30 \%$ energy from fat) and high-dietary fibre $(>3 \mathrm{~g} / \mathrm{MJ})$ diet showed a reduction in the concentration of total cholesterol among young men and women (mean age 24 years) ${ }^{(20)}$. Dragsted et al. ${ }^{(21)}$ also confirmed a significant decrease in the concentration of total cholesterol and LDL

Abbreviation: p-tHcy, plasma total homocysteine.

* Corresponding author: Dr Tonje Holte Stea, fax +47 381413 01, email tonje.h.stea@uia.no 
after providing $600 \mathrm{~g}$ fruit and vegetables per $\mathrm{d}$ to forty-three volunteers for $24 \mathrm{~d}$. Other studies have shown that an increased intake of dietary fibre through vegetables, fruits and wholegrain cereals beneficially affected the lipid profile and the concentration of homocysteine ${ }^{(22,23)}$. Despite the uncertainty whether plasma total homocysteine ( $\mathrm{p}$-tHcy) concentration may be a marker of vascular risk or a causal factor, studies have shown that elevated p-tHcy concentration is associated with an increased risk of $\mathrm{CVD}^{(24,25)}$.

The target group of the present intervention study was men between 18 and 26 years of age, since this group seldom has been the focus of nutrition interventions. The aim of the study was to promote an increased intake of vegetables, fruits and wholegrain bread by focusing on both nutrition information and increased availability of these food items. The effect of the intervention was determined by changes in the intake of selected food items and nutrients. Previously it was reported that this dietary intervention had shown a significant increase in the total intake of vegetables, fruit, berries and fruit juice and wholegrain bread $(P<0.001$ for both) and a reduction in the concentration of $\mathrm{p}$-tHcy $(-10 \% ; P=0.002)$ during the study period ${ }^{(26)}$. The present study further investigated whether the reduction in p-tHcy was associated with changes in folate intake from specific foods, such as vegetables, fruits, juice and wholegrain bread.

\section{Methods}

\section{Study design and participants}

In the intervention group, $541(81.6 \%)$ male recruits (aged 18-26 years) from Værnes military camp agreed to participate in the data collection at baseline and $416(62.7 \%)$ of the recruits completed the entire study period. In the control group, $209(88.2 \%)$ newly enrolled male recruits in the Norwegian Army at Heggelia agreed to participate, but only 105 recruits $(44.4 \%)$ completed the entire study period. Most of the recruits $(89.6 \%)$ who did not complete the entire study had been excluded from military service due to health problems. Supplement use and incomplete datasets were exclusion criteria for the present study. We did not find any sociodemographic differences between participants in the intervention group and the control group that could explain the differences in participation rate.

As Norwegian military service is compulsory for men from the age of 19 years, recruits from different geographical areas and social classes in Norway are randomly distributed to different military camps. The military camps where our data were collected were chosen due to similar levels of physical training during military service. The intervention group was provided with information regarding the benefits of healthy eating habits, as well as easy access to a diet rich in vegetables, fruits and wholegrain bread, and low in fat content. The control group received only practical information regarding the data collection. Blood samples, measurements of body weight and height and information about dietary habits and age were collected during week 1 after the enrolment and after 5 months. The present study was conducted according to the guidelines laid down in the Declaration of Helsinki. All procedures involving human subjects were approved by the Ministry of Health and Care Services, Regional Committee for Medical Research Ethics and the Data Inspectorate. Written informed consent was obtained from the recruits before participation in the study.

\section{Diet}

All recruits were offered free breakfast, lunch, dinner and evening meal in a mess hall or they could choose to buy food in a commercial canteen, both of which were located at the military camps. In the canteen, the recruits had the option to buy fast food, snacks and candies, whereas the availability of fruits and vegetables was very limited. Alternative places to buy food included restaurants, cafeterias and grocery stores located outside Værnes and Heggelia military camps. In the mess hall at Værnes military camp, the commonly used recipes were prepared focusing on vegetables, fruits, wholegrain bread and dietary fat content in line with the Nordic Nutritional Recommendations. The breakfast and evening meals consisted of wholegrain bread and various sandwich spreads. The amount of wholegrain varied between 50 and $100 \%$ in the different bread products. In Norway, folate fortification of bread is not yet allowed. It is also important to notice that refined white flour that is used in Norwegian bread has a high extraction rate (close to $80 \%$ ). For lunch, the recruits could choose between a hot dish, wholegrain bread, various sandwich spreads and a salad bar consisting of some selected vegetables. For dinner they were offered one hot dish and they could also choose to have vegetables (cooked or a mixed salad) and wholegrain bread. In the military mess hall, the recruits selected their own food and portion size. The military chefs were trained in how to prepare and present healthy meals. To avoid differences in the composition of meals due to limited seasonal availability of ingredients, the meals were mainly based on ingredients that were available during all seasons. In the control group at Heggelia military camp, there was no change in the food regimen during military service. This food contained therefore more fat, especially saturated fat, and fewer vegetables and fruits were available, compared with the food offered to the intervention group.

\section{Food diary and calculation of nutrient intake}

A validated food diary developed at the Department of Nutrition, University of Oslo (sef.no/assets/11002260/ vedlegg1_ungkost.PDF) was modified to appropriately reflect the diet served in the military mess hall ${ }^{(27)}$. Frequency and portion sizes of selected food items available in the mess hall were pre-printed choices in the food diary. The food items included vegetables, fruits, wholegrain bread and other cereals, butter, milk products and drinks. When appropriate, the recruits reported their intakes of vegetables, fruits and wholegrain bread in pieces. To assist the recruits and to obtain estimates of the consumption in grams, most of the food items that were printed in the food diary were also preweighed. The estimates are the average of ten weighed pieces. Hot dishes offered at lunch and dinners were included in the diary as open-ended questions in which the recruits filled in a number for the menu of the day. To estimate the consumption of different ingredients included in the hot meals, recipes and estimates of the amounts of the ingredients used in every dish were collected. An average content of 
ingredients in all dishes and in the small, medium, large and extra-large serving sizes was calculated from these data. For registration of side dishes served at lunch and dinner, the recruits stated the size (small, medium, large or extra-large) and number of servings. Serving sizes for these food items were decided with the help from the kitchen staff at the camp and from other Norwegian surveys ${ }^{(28)}$. For determination of serving size, the recruits were asked to refer to photographs and/or weighed portion sizes placed in the military mess during the meals in the food diary period. In addition, two research workers were present at every meal to help the recruits to determine their own portion sizes. This support was given to the recruits in both the intervention group and the control group. The food diary also included questions about how often and what they did eat in the military canteen and/or cafeterias and restaurants outside the military camp.

The nutritional values of recipes and intakes of food and nutrients from the food diaries were calculated using the computer program Mat på Data (version 5.0; Matportalen, Oslo, Norway) and the Norwegian food composition table ${ }^{(29)}$. The average daily consumption for each recruit in the survey period was obtained by adding up their total consumption of selected foods and nutrients and dividing by the number of days during which the subject kept records.

\section{Blood sampling and biochemical analyses}

Blood samples were collected after an overnight fast ( $\geq 12 \mathrm{~h}$ ). The participants were restricted from drinking and using tobacco $12 \mathrm{~h}$ before blood sampling. All blood samples were centrifuged within $4 \mathrm{~h}$ at $2000 \mathrm{~g}$ for $10 \mathrm{~min}$ at $4^{\circ} \mathrm{C}$. Serum and plasma samples were immediately frozen in $1 \mathrm{ml}$ blood collection tubes at $-20^{\circ} \mathrm{C}$ before they were sent to Stavanger University Hospital, Norway and stored at $-70^{\circ} \mathrm{C}$ until analysed. Total serum cholesterol, TAG and HDL were measured with enzymic procedures (Roche, Mannheim, Germany) at Stavanger University Hospital. The concentration of LDL was estimated by using the Friedewald formula (total cholesterol $-\mathrm{HDL}-0.46 \times \mathrm{TAG})^{(30)}$. The plasma concentration of tHcy was determined by $\mathrm{HPLC}^{(31)}$. The $\mathrm{CV}$ for the measured variables were $<0.3 \%$ for total cholesterol, $<0.7 \%$ for TAG, $<2 \%$ for HDL and $6.4 \%$ for $\mathrm{p}$-tHcy.

\section{Statistical analyses}

Changes in dietary intakes of food and nutrients are presented as means and corresponding 95\% CI (Tables 1-3). A multiple regression model calculated the intervention effects as the ratio of means of the intervention group relative to the control group, adjusted for baseline concentrations ${ }^{(32)}$ (Fig. 1 and Tables 1-3). The regression models were checked for normality of residuals, linearity and homogeneity of variance. A multiple regression model was also used to calculate the predictors of changes in p-tHcy concentration (Table 4). Because of skewed distribution, change in the concentration of p-tHcy (dependent variable) was transformed to the natural logarithm before fitting the regression model. All statistical analyses were conducted with SPSS (version 15.0; SPSS Inc., Chicago, IL, USA).

\section{Results}

Baseline characteristics of those participants who completed the entire study showed a significantly lower BMI in the intervention group than in the control group (Table $1 ; P=0.004$ ). The concentrations of p-tHcy and serum lipids, however, were not significantly different between the groups.

Changes in the dietary intake of energy from carbohydrates, proteins and different types of fat are listed in Table 2. The results showed a significantly increased energy intake in the intervention group compared with the control group $(P=0.002)$. The percentage of energy derived from carbohydrates and PUFA increased in the intervention group compared with the control group $(P<0.001$ for both). On the contrary, a significantly reduced percentage of energy was derived from total fat, SFA, MUFA and proteins in the intervention group compared with the control group $(P<0.001$ for all). The dietary intervention showed no effect on the serum concentration of total cholesterol, LDL and HDL in serum (data not shown). A minor increase in the concentration of TAG was shown in both groups at the end of the study period. The increase in the concentration of TAG, however, was significantly higher in the control group compared with the intervention group $(P=0 \cdot 045)$.

Baseline registration showed a total intake of vegetables, fruits, berries and juice of $434 \mathrm{~g} / \mathrm{d}$ in the intervention group and $450 \mathrm{~g} / \mathrm{d}$ in the control group. The total consumption of

Table 1. Baseline characteristics of the study participants who completed the entire study (Mean values and $95 \%$ confidence intervals)

\begin{tabular}{|c|c|c|c|c|c|}
\hline \multirow[b]{2}{*}{ Variable } & \multicolumn{2}{|c|}{ Intervention group } & \multicolumn{2}{|c|}{ Control group } & \multirow[b]{2}{*}{$P$} \\
\hline & Mean & $95 \% \mathrm{Cl}$ & Mean & $95 \% \mathrm{Cl}$ & \\
\hline Participants $(n)$ & \multicolumn{2}{|c|}{414} & \multicolumn{2}{|c|}{91} & \\
\hline Age (years) & $19 \cdot 6$ & $19 \cdot 4,19.7$ & $19 \cdot 3$ & $19 \cdot 1,19 \cdot 5$ & 0.059 \\
\hline BMI $\left(\mathrm{kg} / \mathrm{m}^{2}\right)$ & 23.3 & $23 \cdot 0,23 \cdot 6$ & 24.5 & $23 \cdot 8,25 \cdot 3$ & 0.004 \\
\hline $\mathrm{tHcy}(\mu \mathrm{mol} / \mathrm{l})$ & $11 \cdot 3^{*}$ & $10 \cdot 9,11 \cdot 7$ & $11 \cdot 5^{\star}$ & $10 \cdot 8,12 \cdot 3$ & 0.830 \\
\hline Total cholesterol $(\mathrm{mmol} / \mathrm{l})$ & $3 \cdot 8$ & $3 \cdot 8,3 \cdot 9$ & 3.9 & $3 \cdot 7,4 \cdot 0$ & 0.770 \\
\hline TAG (mmol/l) & $0 \cdot 7$ & $0 \cdot 7,0.7$ & 0.7 & $0 \cdot 6,0.8$ & 0.655 \\
\hline $\mathrm{HDL}(\mathrm{mmol} / \mathrm{l})$ & 1.3 & $1 \cdot 3,1 \cdot 4$ & 1.3 & $1 \cdot 3,1 \cdot 4$ & 0.686 \\
\hline LDL (mmol/l) & $2 \cdot 2$ & $2 \cdot 1,2 \cdot 2$ & $2 \cdot 2$ & $2 \cdot 1,2 \cdot 4$ & 0.575 \\
\hline
\end{tabular}

tHcy, plasma total homocysteine.

${ }^{\star}$ Concentration of $\mathrm{tHcy}$ is presented as the geometric mean. 
Table 2. Changes in energy intake during the intervention study† (Mean values and $95 \%$ confidence intervals)

\begin{tabular}{|c|c|c|c|c|c|c|c|}
\hline & \multicolumn{2}{|c|}{ Baseline } & \multicolumn{2}{|c|}{ After 5 months } & \multirow[b]{2}{*}{ Change (\%) } & \multicolumn{2}{|c|}{ Intervention effect $\ddagger$} \\
\hline & Mean & $95 \% \mathrm{Cl}$ & Mean & $95 \% \mathrm{Cl}$ & & Mean & $95 \% \mathrm{Cl}$ \\
\hline \multicolumn{8}{|l|}{ Energy (kJ) } \\
\hline Intervention group & 8676 & 8354,8997 & 9349 & 8998,9700 & $+7 \cdot 8$ & & \\
\hline Control group & 10905 & 10295,11514 & 9234 & 8650,9818 & $-15 \cdot 3$ & $1006^{*}$ & 377,1635 \\
\hline \multicolumn{8}{|l|}{ Fat (\% energy) } \\
\hline Intervention group & $33 \cdot 1$ & $32 \cdot 2,34 \cdot 0$ & $27 \cdot 8$ & $26 \cdot 8,28 \cdot 9$ & $-16 \cdot 0$ & & \\
\hline Control group & $36 \cdot 3$ & $35 \cdot 0,37 \cdot 7$ & $33 \cdot 8$ & $32 \cdot 4,35 \cdot 1$ & -6.9 & $-3 \cdot 4^{\star \star}$ & $-5 \cdot 1,-1 \cdot 7$ \\
\hline \multicolumn{8}{|l|}{ SFA (\% energy) } \\
\hline Intervention group & $13 \cdot 0$ & $12 \cdot 7,13 \cdot 3$ & $9 \cdot 6$ & $9 \cdot 3,10 \cdot 0$ & $-26 \cdot 1$ & & \\
\hline Control group & 13.9 & $13.4,14.4$ & $13 \cdot 5$ & $13 \cdot 0,14.0$ & -2.9 & $-2 \cdot 9^{\star \star}$ & $-3 \cdot 6,-2 \cdot 3$ \\
\hline \multicolumn{8}{|l|}{ MUFA (\% energy) } \\
\hline Intervention group & 9.5 & $9 \cdot 3,9 \cdot 8$ & $6 \cdot 5$ & $6 \cdot 3,6 \cdot 7$ & $-31 \cdot 6$ & & \\
\hline Control group & $10 \cdot 8$ & $10 \cdot 4,11 \cdot 3$ & $10 \cdot 1$ & $9 \cdot 7,10 \cdot 5$ & -6.5 & $-3 \cdot 0^{\star *}$ & $-3.4,-2.5$ \\
\hline \multicolumn{8}{|l|}{ PUFA (\% energy) } \\
\hline Intervention group & 8.0 & $7 \cdot 6,8 \cdot 5$ & $9 \cdot 1$ & $8 \cdot 6,9.6$ & $+13 \cdot 7$ & & \\
\hline Control group & 8.7 & $8 \cdot 0,9 \cdot 3$ & 8.0 & $7 \cdot 3,8 \cdot 7$ & -8.0 & $1.5^{\star \star}$ & $0.7,2.2$ \\
\hline \multicolumn{8}{|c|}{ Carbohydrates (\% energy) } \\
\hline Intervention group & $51 \cdot 1$ & $50 \cdot 2,52 \cdot 0$ & $58 \cdot 3$ & $57 \cdot 4,59 \cdot 3$ & $+14 \cdot 1$ & & \\
\hline Control group & $47 \cdot 4$ & $46 \cdot 1,48 \cdot 8$ & $50 \cdot 6$ & $49 \cdot 2,52 \cdot 0$ & $+6 \cdot 7$ & $5 \cdot 0^{* *}$ & $3 \cdot 3,6 \cdot 7$ \\
\hline \multicolumn{8}{|l|}{ Protein (\% energy) } \\
\hline Intervention group & $15 \cdot 8$ & $15 \cdot 5,16 \cdot 1$ & $13 \cdot 8$ & $13 \cdot 5,14 \cdot 1$ & $-12 \cdot 7$ & & \\
\hline Control group & $16 \cdot 2$ & $15 \cdot 6,16 \cdot 9$ & $15 \cdot 6$ & $15 \cdot 0,16 \cdot 2$ & -3.7 & $-1 \cdot 4^{\star \star}$ & $-2 \cdot 0,-0.9$ \\
\hline
\end{tabular}

The effect of the intervention was significant: ${ }^{\star} P<0.05,{ }^{\star \star} P<0.001$.

†Intervention group, $n$ 376; control group, $n 105$.

$\ddagger$ The intervention effect was calculated as the mean of the intervention group relative to the control group, adjusted for baseline values.

wholegrain bread at baseline was 175 and $188 \mathrm{~g} / \mathrm{d}$ in the intervention and control groups, respectively. After the 5-month dietary intervention, the total intake of vegetables, fruits, berries and juice increased to $538 \mathrm{~g} / \mathrm{d}(+24 \%)$ in the intervention group and $478 \mathrm{~g} / \mathrm{d}(+6 \%)$ in the control group (intervention effect; $P=0 \cdot 014)$. The consumption of wholegrain bread increased to $225 \mathrm{~g} / \mathrm{d}(+29 \%)$ and decreased to $180 \mathrm{~g} / \mathrm{d}$ $(-4.2 \%)$ in the intervention and control groups, respectively (intervention effect; $P<0.001$ ). The estimated total intake of dietary fibre and folate increased significantly in the intervention group compared with the control group during the study period (Table 3; $P<0.001$ for both). A minor but significant reduction was shown in the dietary intake of vitamin $B_{2}$ (riboflavin) in the intervention group compared with the control group $(P=0 \cdot 018)$. A positive correlation, however, was shown between changes in estimated total intake of dietary fibre and vitamin $\mathrm{B}_{2}\left(R_{\mathrm{p}} 0.502\right)$, dietary fibre and folate $\left(R_{\mathrm{p}} 0.720\right)$ and folate and vitamin $\mathrm{B}_{2}\left(R_{\mathrm{p}} 0.652\right)$ during the study period $(P<0 \cdot 001$ for all).

The total intake of estimated dietary fibre from vegetables, fruits, berries and juice increased from $4.4 \mathrm{~g} / \mathrm{d}$ to $4.9 \mathrm{~g} / \mathrm{d}$ in the intervention group and decreased from $4.4 \mathrm{~g} / \mathrm{d}$ to $3.4 \mathrm{~g} / \mathrm{d}$ in the control group. At the same time, the total intake of estimated dietary fibre from wholegrain bread increased from $10.2 \mathrm{~g} / \mathrm{d}$ to $13.2 \mathrm{~g} / \mathrm{d}$ in the intervention group and from $11.1 \mathrm{~g} / \mathrm{d}$ to $11.6 \mathrm{~g} / \mathrm{d}$ in the control group (Fig. 1). Thus, a significant effect of the intervention was shown in the relative contribution of dietary fibre from both vegetables, fruits, berries and juice $(P<0 \cdot 001)$ and wholegrain bread $(P=0 \cdot 001)$. Furthermore, the study showed a significant increase in the estimated folate intake

Table 3. Changes in dietary intake of fibre, folate and vitamin $B_{2}$ during the intervention study $\dagger$

(Mean values and $95 \%$ confidence intervals)

\begin{tabular}{|c|c|c|c|c|c|c|c|}
\hline & \multicolumn{2}{|c|}{ Baseline } & \multicolumn{2}{|c|}{ After 5 months } & \multirow[b]{2}{*}{ Change (\%) } & \multicolumn{2}{|c|}{ Intervention effect $\ddagger$} \\
\hline & Mean & $95 \% \mathrm{Cl}$ & Mean & $95 \% \mathrm{Cl}$ & & Mean & $95 \% \mathrm{Cl}$ \\
\hline \multicolumn{8}{|l|}{ Fibre $(g / d)$} \\
\hline Intervention group & $24 \cdot 7$ & $23 \cdot 4,26 \cdot 0$ & $31 \cdot 1$ & $29 \cdot 6,32 \cdot 6$ & +25.9 & & \\
\hline Control group & 24.5 & $22 \cdot 8,26 \cdot 2$ & $24 \cdot 2$ & $22 \cdot 3,26 \cdot 0$ & $-1 \cdot 2$ & $5 \cdot 9^{* *}$ & $3 \cdot 6,8 \cdot 3$ \\
\hline \multicolumn{8}{|l|}{ Folate $(\mu \mathrm{g} / \mathrm{d})$} \\
\hline Intervention group & $196 \cdot 3$ & $188 \cdot 4,204 \cdot 2$ & $257 \cdot 7$ & $248 \cdot 5,266 \cdot 9$ & $+31 \cdot 3$ & & \\
\hline Control group & $189 \cdot 9$ & $179 \cdot 4,200 \cdot 5$ & $209 \cdot 8$ & $196 \cdot 0,223.5$ & +10.5 & $31 \cdot 0^{\star \star}$ & $15 \cdot 3,46 \cdot 7$ \\
\hline \multicolumn{8}{|l|}{ Vitamin $B_{2}(\mathrm{mg} / \mathrm{d})$} \\
\hline Intervention group & 1.54 & $1.45,1.60$ & 1.49 & $1.43,1.56$ & $-2 \cdot 7$ & & \\
\hline Control group & 1.81 & $1.69,1.92$ & 1.76 & $1.64,1.92$ & -2.4 & $-0 \cdot 1^{*}$ & $-0.3,0.0$ \\
\hline
\end{tabular}

The effect of the intervention was significant: ${ }^{\star} P<0.05$, ${ }^{\star \star} P<0.001$.

†Intervention group, $n$ 376; control group, $n 105$.

$\ddagger$ The intervention effect was calculated as the mean of the intervention group relative to the control group, adjusted for baseline values. 


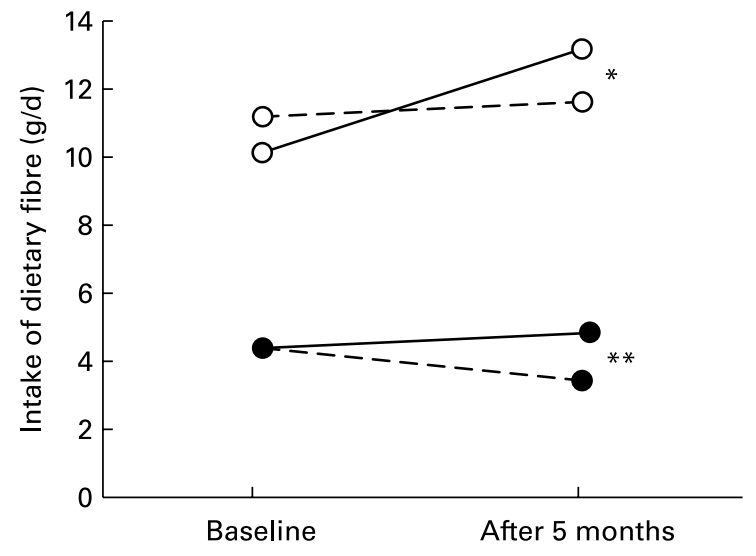

Fig. 1. Changes in intake of dietary fibre from wholegrain bread and vegetables, fruits, berries and juice in the intervention study in the intervention ( $n$ 376) and control ( $n$ 105) groups. (-O-), Wholegrain bread, intervention group; (--O--), wholegrain bread, control group; (--), sum of vegetables, fruits, berries and juice, intervention group; (--๑- -), sum of vegetables, fruits, berries and juice, control group. The intervention effect was calculated as the mean of the intervention group relative to the control group, adjusted for baseline values. The effect of the intervention was significant: ${ }^{\star} P=0.001$, ${ }^{\star \star} P<0.001$

from different food groups, including vegetables, fruits and wholegrain bread (intervention effect, $P<0.001$ for all; Table 4). The estimated folate intake from different food groups, both at baseline and after the 5-month intervention period, showed that wholegrain bread was the most important single contributor to total folate intake.

Furthermore, we investigated the association between changes in the concentration of p-tHcy and changes in absolute intakes of major food groups (fruits, vegetables, juice, wholegrain bread, milk products and potatoes) during the intervention study. There was an inverse relationship between the concentration of p-tHcy and the intake of wholegrain bread after adjustment for changes in BMI, physical fitness and the concentration of total cholesterol during the study period (data not shown; $P=0 \cdot 034$ ). A significant association between the estimated increase in dietary intake of folate from wholegrain bread and the decrease in p-tHcy concentration was also shown $(P=0.010$; Table 5). Changes in folate intake from fruits and vegetables were not significantly related to the changes in p-tHcy concentration. The variables included in the multivariate linear regression analysis explained in combination $16.2 \%$ of the variance in the change of $\mathrm{p}$-tHcy concentration during the study period.

\section{Discussion}

The present study was performed to improve dietary habits and reduce cardiovascular risk factors among young men after a 5-month dietary intervention with a focus on vegetables, fruit and wholegrain bread. Despite the random distribution of recruits to different military camps, baseline results for those who completed the entire study showed a significantly lower BMI among recruits in the intervention group compared with the control group. Furthermore, the present study showed a significantly lower energy intake in the intervention group compared with the control group at baseline. However, there were no statistical differences in the concentration of p-tHcy and serum lipids.

A very limited number of studies have focused on reducing modifiable risk factors among young male adults, as this target group has been considered a difficult group to reach when promoting a healthy lifestyle. In the present study, we have demonstrated an inverse relationship between the estimated change in folate intake from wholegrain bread and the

Table 4. Changes in relative contribution of different food groups to total folate intake†

(Mean values and $95 \%$ confidence intervals)

\begin{tabular}{|c|c|c|c|c|c|c|c|}
\hline & \multicolumn{2}{|c|}{ Baseline $(\mu \mathrm{g} / \mathrm{d})$} & \multicolumn{2}{|c|}{ After 5 months $(\mu \mathrm{g} / \mathrm{d})$} & \multirow[b]{2}{*}{ Change (\%) } & \multicolumn{2}{|c|}{ Intervention effect $\ddagger$} \\
\hline & Mean & $95 \% \mathrm{Cl}$ & Mean & $95 \% \mathrm{Cl}$ & & Mean & $95 \% \mathrm{Cl}$ \\
\hline \multicolumn{8}{|l|}{ Vegetables } \\
\hline Intervention group & $16 \cdot 4$ & $14 \cdot 7,18 \cdot 0$ & $19 \cdot 6$ & $17 \cdot 5,21 \cdot 8$ & +19.5 & & \\
\hline Control group & $23 \cdot 6$ & $19 \cdot 2,28 \cdot 1$ & $11 \cdot 3$ & $8 \cdot 6,14 \cdot 0$ & $-52 \cdot 1$ & $10 \cdot 1^{\star *}$ & $5 \cdot 9,14 \cdot 2$ \\
\hline \multicolumn{8}{|l|}{ Fruits } \\
\hline Intervention group & $8 \cdot 2$ & $7 \cdot 1,9 \cdot 3$ & $16 \cdot 2$ & $14 \cdot 4,18 \cdot 0$ & +97.6 & & \\
\hline Control group & 8.4 & $5 \cdot 6,11 \cdot 2$ & $7 \cdot 6$ & $5 \cdot 4,9 \cdot 7$ & -9.5 & $8 \cdot 4^{\star \star}$ & $4 \cdot 7,12 \cdot 1$ \\
\hline \multicolumn{8}{|l|}{ Juice } \\
\hline Intervention group & 13.9 & $11 \cdot 6,16 \cdot 2$ & $22 \cdot 9$ & $19 \cdot 6,26 \cdot 2$ & $+64 \cdot 7$ & & \\
\hline Control group & $15 \cdot 9$ & $11 \cdot 7,20 \cdot 1$ & $17 \cdot 8$ & $13 \cdot 4,22 \cdot 2$ & +11.9 & $5 \cdot 2$ & $-1 \cdot 7,12 \cdot 1$ \\
\hline \multicolumn{8}{|l|}{ Wholegrain bread } \\
\hline Intervention group & 44.2 & $42 \cdot 3,46 \cdot 1$ & $57 \cdot 3$ & $55 \cdot 0,59 \cdot 6$ & $+29 \cdot 6$ & & \\
\hline Control group & $48 \cdot 7$ & $44 \cdot 9,52 \cdot 5$ & $47 \cdot 9$ & $43 \cdot 7,52 \cdot 1$ & -1.6 & $12 \cdot 0^{\star *}$ & $7 \cdot 4,16 \cdot 7$ \\
\hline \multicolumn{8}{|l|}{ VF } \\
\hline Intervention group & 41.9 & $38 \cdot 5,45 \cdot 4$ & $61 \cdot 8$ & $56 \cdot 9,66 \cdot 6$ & +47.5 & & \\
\hline Control group & 49.9 & $42 \cdot 1,57 \cdot 6$ & $40 \cdot 9$ & $34 \cdot 3,47 \cdot 4$ & $-18 \cdot 0$ & $22 \cdot 1^{\star *}$ & $12 \cdot 4,31 \cdot 8$ \\
\hline \multicolumn{8}{|l|}{ Total } \\
\hline Intervention group & $82 \cdot 8$ & $78 \cdot 5,87 \cdot 0$ & $114 \cdot 3$ & $108 \cdot 7,119 \cdot 8$ & +38.0 & & \\
\hline Control group & $97 \cdot 0$ & $87 \cdot 3,106 \cdot 7$ & $84 \cdot 8$ & $76 \cdot 6,93 \cdot 1$ & $-12 \cdot 6$ & $35 \cdot 7^{\star \star}$ & $24 \cdot 9,46 \cdot 5$ \\
\hline
\end{tabular}

VF, sum of vegetables, fruits, berries and juice; Total, sum of vegetables, fruit, juice and wholegrain bread.

** The effect of the intervention was significant $(P<0.001)$.

†Intervention group, $n$ 376; control group, $n 105$

$\ddagger$ The intervention effect was calculated as the mean folate intake of the intervention group relative to the control group, adjusted for baseline values. 
Table 5. Unstandardised multiple linear regression coefficients as predictors of changes in plasma total homocysteine (p-tHcy) concentration during the intervention study for 340 subjects $^{*}$

\begin{tabular}{|c|c|c|c|c|}
\hline \multirow[b]{2}{*}{ Predictor variables } & \multicolumn{2}{|c|}{ Univariate linear regression } & \multicolumn{2}{|c|}{ Multivariate linear regression† } \\
\hline & B unstandardised & $P$ & $\mathrm{~B}_{\text {unstandardised }}$ & $P$ \\
\hline \multicolumn{5}{|l|}{ Changes in } \\
\hline Folate intake from fruits $(\mu \mathrm{g} / \mathrm{d})$ & 0.000 & 0.258 & 0.000 & 0.556 \\
\hline Folate intake from vegetables $(\mu \mathrm{g} / \mathrm{d})$ & 0.000 & 0.651 & 0.000 & 0.844 \\
\hline Folate intake from juice $(\mu \mathrm{g} / \mathrm{d})$ & 0.000 & 0.528 & 0.000 & 0.211 \\
\hline Folate intake from wholegrain bread $(\mu \mathrm{g} / \mathrm{d})$ & -0.001 & 0.007 & -0.001 & 0.010 \\
\hline Total folate intake $(\mu \mathrm{g} / \mathrm{d})$ & -0.003 & 0.031 & & \\
\hline
\end{tabular}

* The dependent variable $p$-tHcy is $\log _{\mathrm{e}}$-transformed before comparison. The dependent variable ( $\mathrm{p}$-tHcy) and predictor variables are computed as follow-up concentrations minus baseline concentrations.

†Adjusted for changes in BMI, physical fitness and concentration of total cholesterol during the intervention study.

concentration of p-tHcy. Fat intake, particularly intake of SFA, has been positively associated with serum cholesterol and mortality from CVD in many epidemiological and longitudinal studies ${ }^{(33,34)}$. The present dietary intervention resulted in an estimated reduction in energy consumption from fat, more in line with the recommendations from the Nordic Council of Ministers ${ }^{(35)}$, but this was not confirmed by changes in the concentration of serum lipids. On the contrary, marginal increases in the concentration of TAG were shown in both the intervention group and the control group, but significantly higher in the latter group. Durstine et al. ${ }^{(36)}$ have demonstrated that an increase in physical capacity was associated with a reduced concentration of TAG. Recently published data from the present study showed that the intervention group significantly improved the result from the $3000 \mathrm{~m}$ run compared with the control group ${ }^{(26)}$. Thus, differences in physical exercise among the recruits may also have contributed to differences in the concentration of TAG at the end of the study period.

The baseline intake of vegetables, fruits, berries and juice and wholegrain bread was fairly low among all participants in the present study. The present dietary intervention, based on increased nutrition information and increased availability of vegetables, fruits and wholegrain bread, resulted in an increased consumption of both vegetables, fruits, berries and juice and wholegrain bread. These changes in dietary intake indicate that the main aim of our dietary intervention was achieved.

Another focus of the present study was to investigate baseline intakes of folate, vitamin $\mathrm{B}_{2}$ and dietary fibre and changes in intake of these nutrients during the study period. Baseline results showed a low daily intake of folate in both groups, whereas the mean intake of vitamin $\mathrm{B}_{2}$ was low in the intervention group and adequate in the control group compared with the Nordic nutritional recommendations for healthy male adults ${ }^{(35)}$. Compared with the present results, a higher intake of vitamin $B_{2}$ was shown in both Norkost II $(2.3 \mathrm{mg} / \mathrm{d})$ and among Irish young male adults $(2.2 \mathrm{mg} / \mathrm{d})$ in the Young Hearts Project ${ }^{(37,38)}$. The latter study also showed a considerable higher dietary intake of folate $(369 \mu \mathrm{g} / \mathrm{d})$ among men aged 20 25 years $^{(37)}$ than what was found in the present study. The intake of dietary fibre, which has been shown to be inversely associated with the risk of CHD in both men and women ${ }^{(39)}$, should range between 25 and $35 \mathrm{~g} / \mathrm{d}$ according to the Nordic nutritional recommendations ${ }^{(35)}$. In the present study, the estimated baseline intake of dietary fibre ( 24.5 and $24.7 \mathrm{~g} / \mathrm{d}$ for the control and intervention groups, respectively) corresponded to the results from Norkost $\mathrm{II}^{(38)}$ which showed a lower dietary intake of fibre $(24 \mathrm{~g} / \mathrm{d})$ among men between 20 and 29 years old than recommended $^{(35)}$.

Our intervention study resulted in a significant increase in the estimated total intake of both dietary fibre and folate, which is an outcome of a higher consumption of vegetables, fruits and wholegrain bread. The present study also showed that the increased consumption of wholegrain bread contributed more to the estimated increase in intake of both dietary fibre and folate than the increased consumption of fruits and vegetables. Our findings were in agreement with previous studies, which have demonstrated that wholegrain bread is quantitatively one of the most important contributors to total intake of dietary fibre and folate among both men and women in Norway ${ }^{(38,40)}$. Despite a minor reduction in the estimated intake of vitamin $B_{2}$ on a group level, a positive correlation was shown between the changes in intake of dietary fibre, folate and vitamin $B_{2}$ on an individual level. Thus, the recruits who increased their intake of vitamin $B_{2}$ seemed to have a greater increase in the intake of dietary fibre and folate than the recruits who decreased their intake of vitamin $\mathrm{B}_{2}$ during the study period.

Previously reported results from this dietary intervention have demonstrated a significant relationship between an increased concentration of folate and a reduction in the concentration of p-tHcy during the study period ${ }^{(26)}$. Thus, we expect that the present results may be related to an increased intake of folate from unfortified foods. Few intervention studies have focused on the relationship between changes in folate intake from different food groups and the effect on p-tHcy concentration. Winkels et al. ${ }^{(41)}$ demonstrated that wholegrain bread fortified with folic acid significantly increased folate status and reduced p-tHcy concentrations. In Norway, however, fortification of foods with folate is not allowed. The results from the present study showed a weak, but statistically significant, relationship between the estimated increase in folate intake from wholegrain bread, which had not been fortified with folic acid, and the decreased concentration of p-tHcy. On the other hand, changes in the estimated intake of folate from vegetables, fruits or juice did not show any significant relationship with changes in the concentration of p-tHcy, respectively. A possible explanation for the lack of association between p-tHcy concentration and estimated 
intake of folate from fruits and juices is the low bioavailability of folate from these food sources ${ }^{(42,43)}$. Similar to the present results, large cross-sectional studies have not shown any association between the concentration of tHcy in plasma and serum and the folate intake from orange juice or fruits ${ }^{(42,44)}$. The Framingham Heart Study, however, showed that the intake of green leafy vegetables was related to lower concentrations of p-tHcy ${ }^{(4)}$. Ganji \& $\mathrm{Kafai}^{(42)}$ also demonstrated an inverse relationship between the serum concentration of tHcy and intake of cruciferous vegetables, but not with intake of other vegetables or wholegrain bread. The latter study suggested that the lack of association between serum concentration of tHcy and total intake of vegetables was due to the variable B-vitamin content in different vegetables ${ }^{(42)}$.

The lack of association between the p-tHcy concentration and the estimated intake of folate from vegetables in the present study may also be explained by a reduced concentration of folate due to food processing. This is in accordance with the results from a previous study which showed a decreased retention of folate in vegetables caused by different processes used in modern large-scale service systems, including the military service system ${ }^{(45)}$.

\section{Conclusion}

Our data showed that a simple, but targeted, nutrition intervention programme based on nutritional information and increased availability of vegetables, fruits and wholegrain bread was effective in modifying food intake of healthy young males. An increased intake of vegetables, fruits and wholegrain bread was associated with beneficial modifications in nutrient intake, with wholegrain bread as the best source of folate. A small, but significant, association was demonstrated between an increased intake of folate from wholegrain bread and a decreased concentration of p-tHcy. There is a debate on the positive and negative aspects of folic acid fortification in many countries. The present study showed evidence of an alternative policy approach based on nutrition education and a food-based alternative to supplementation and/or folic acid fortification.

\section{Acknowledgements}

The present study was supported by grants from the Norwegian Research Council (grant no. 154320).

W. F. and M. W. were responsible for the study concept and design. T. H. S. and S. U. acquired the data. M. A. M. organised p-tHcy analyses and helped to interpret the results. T. H. S. was responsible for the interpretation of data, statistical analysis and drafting of the manuscript. M. W. and M. A. M. were involved in the critical revision of the manuscript for important intellectual content.

The authors want to thank the personnel at The Norwegian Home Guard Training Centre Vaernes for helping to organise the data collections. The authors are grateful to Elin Skaarland Frøyland and Marte Råberg for their excellent cooperation during data processing.

There were no conflicts of interest.

\section{References}

1. Klepp K-I, Halvorsen M, Bjørneboe GEA, et al. (1996) Evaluating 'Food in the Schools' - changes over time in reported meal frequency among Norwegian school children. Scand $J$ Nutr 40, 113-116.

2. Andersen LF, Øverby N \& Lillegaard IT (2004) Intake of fruit and vegetables among Norwegian children and adolescents. Tidsskr Nor Laegeforen 124, 1396-1398.

3. Joffe M \& Robertson A (2001) The potential contribution of increased vegetable and fruit consumption to health gain in the European Union. Public Health Nutr 4, 893-901.

4. Thane CW, Jones AR, Stephen AM, et al. (2005) Whole-grain intake of British young people aged 4-18 years. Br J Nutr 94, 825-831.

5. World Health Organization (2003) Diet, Nutrition and the Prevention of Chronic Diseases. Joint WHO/FAO Expert Consultation. WHO Technical Report Series no. 916. Geneva: WHO.

6. World Health Organization (2006) Physical Activity and Health in Europe: Evidence for Action. Copenhagen: WHO.

7. Biró L, Regöly-Mérei A, Nagy K, et al. (2007) Dietary habits of school children: representative survey in metropolitan elementary schools. Part two. Ann Nutr Metab 51, 454-460.

8. Chu NF, Rimm EB, Wang DJ, et al. (1998) Clustering of cardiovascular disease risk factors among obese schoolchildren: the Taipei Children Heart Study. Am J Clin Nutr 67, 1141-1146.

9. Gidding SS (2006) Cardiovascular risk factors in adolescents. Curr Treat Options Cardiovasc Med 8, 269-275.

10. Kvaavik E, Lien N, Tell G, et al. (2005) Psychosocial predictors of eating habits among adults in their mid-30s: The Oslo Youth Study follow-up 1991-1999. Int J Behav Nutr Phys Act 2, 9.

11. Pomerleau J, Lock K, Knai C, et al. (2005) Interventions designed to increase adult fruit and vegetable intake can be effective: a systematic review of the literature. J Nutr $\mathbf{1 3 5}$, 2486-2495.

12. Hjermann I (1988) Strategies for dietary and anti-smoking advice: practical experiences from the Oslo Study. Drugs 36, $105-109$

13. Manios Y, Moschonis G, Katsaroli I, et al. (2007) Changes in diet quality score, macro- and micronutrients intake following a nutrition education intervention in postmenopausal women. J Hum Nutr Diet 20, 126-131.

14. Frazao E \& Allshouse J (2003) Strategies for intervention: commentary and debate. J Nutr 133, Suppl. 3, S844-S847.

15. Heitmann BL \& Lissner L (2003) Controversies in Nutritional Epidemiology: Do We Have Evidence that Obesity Prevention Works? A Debate on Design and Methodologies, 9th European Nutrition Conference, Rome, Italy. Nutr Metab 47, 328.

16. Van Duyn MA, Kristal AR, Dodd K, et al. (2001) Association of awareness, intrapersonal and interpersonal factors, and stage of dietary change with fruit and vegetable consumption: a national survey. Am J Health Promot 16, 69-78.

17. Baranowski T, Baranowski J, Cullen KW, et al. (2002) 5 a day Achievement Badge for African-American Boy Scouts: pilot outcome results. Prev Med 34, 353-363.

18. Perry CL, Bishop DB, Taylor GL, et al. (2004) A randomized school trial of environmental strategies to encourage fruit and vegetable consumption among children. Health Educ Behav 31, 65-76.

19. Parker L \& Fox A (2001) The Peterborough Schools Nutrition Project: a multiple intervention programme to improve schoolbased eating in secondary schools. Public Health Nutr 4, $1221-1228$.

20. Sandström B, Marckmann P \& Bindeslev N (1992) An eightmonth controlled study of a low-fat high-fibre diet: effects on 
blood lipids and blood pressure in healthy young subjects. Eur $J$ Clin Nutr 46, 95-109.

21. Dragsted LO, Krath B, Ravn-Haren G, et al. (2006) Biological effects of fruit and vegetables. Proc Nutr Soc 65, 61-67.

22. Erkkilä AT \& Lichtenstein AH (2006) Fiber and cardiovascular disease risk: how strong is the evidence? J Cardiovasc Nurs $\mathbf{2 1}$, $3-8$.

23. Lairon D, Arnault N, Bertrais S, et al. (2005) Dietary fiber intake and risk factors for cardiovascular disease in French adults. Am J Clin Nutr 82, 1185-1194.

24. Chambers JC, Obeid OA, Refsum H, et al. (2000) Plasma homocysteine concentrations and risk of coronary heart disease in UK Indian Asian and European men. Lancet 355, 523-527.

25. Homocysteine Studies Collaboration (2002) Homocysteine and risk of ischemic heart disease and stroke: a meta-analysis. JAMA 288, 2015-2022.

26. Stea TH, Mansoor MA, Wandel M, et al. (2008) Changes in predictors and status of homocysteine in young male adults after a dietary intervention with vegetables, fruits and bread. Eur J Nutr 47, 201-209.

27. Uglem S, Frølich W, Stea TH, et al. (2007) Correlates of vegetable consumption among young men in the Norwegian National Guard. Appetite 48, 46-53.

28. Øverby NC, Lillegaard IT, Johansson L, et al. (2003) High intake of added sugar among Norwegian children and adolescents. Public Health Nutr 7, 285-293.

29. The Norwegian Food Safety Authority Directorate for Health and Social Affairs and the University of Oslo (2006) The Norwegian Food Composition Table. http://www. norwegianfoodcomp.no/

30. Friedewald WT, Levy RI \& Fredrickson DS (1972) Estimation of the concentration of low-density lipoprotein cholesterol in plasma, without use of the preparative ultracentrifuge. Clin Chem 18, 499-502.

31. Mansoor MA, Svardal AM \& Ueland PM (1992) Determination of the in vivo redox status of cysteine, cysteinglycine, homocysteine, and glutathione in human plasma. Anal Biochem 200, 218-229.

32. Vickers AJ \& Altman DG (2001) Statistics notes: analysing controlled trials with baseline and follow up measurements. BMJ 323, 1123-1124.

33. Caggiula AW \& Mustad VA (1997) Effects of dietary fat and fatty acids on coronary artery disease risk and total and lipoprotein cholesterol concentrations: epidemiologic studies. Am J Clin Nutr 65, Suppl. 5, S1597-S1610.
34. Kromhout D, Menotti A, Bloemberg B, et al. (1995) Dietary saturated and trans fatty acids and cholesterol and 25-year mortality from coronary heart disease: the Seven Countries Study. Prev Med 24, 308-315.

35. Nordic Council of Ministers (2005) Nordic Nutrition Recommendations 2004 - Integrating Nutrition and Physical Activity, 4th ed. Copenhagen: Nordic Council of Ministers.

36. Durstine JL, Grandjean PW, Davis PG, et al. (2001) Blood lipid and lipoprotein adaptations to exercise: a quantitative analysis. Sports Med 31, 1033-1062.

37. Gallagher AM, Robson PJ, Livingstone MB, et al. (2006) Tracking of energy and nutrient intakes from adolescence to young adulthood: the experiences of the Young Hearts Project, Northern Ireland. Public Health Nutr 9, 1027-1034.

38. Johansson L \& Solvoll K (1999), Norkost 1997. Landsomfattende Kostholdsunders $\phi k e l s e$ Blant Menn og Kvinner i Alderen 16-79 ̊r (Norkost 1997. Nationwide Dietary Survey Among Men and Women aged 16-79 Years), Rapport nr. 2. Oslo: Statens Råd for Ernæring og Fysisk Aktivitet.

39. Pereira MA, O’Reilly E, Augustsson K, et al. (2004) Dietary fiber and risk of coronary heart disease: a pooled analysis of cohort studies. Arch Intern Med 164, 370-376.

40. Brevik A, Vollset SE, Tell GS, et al. (2005) Plasma concentration of folate as a biomarker for the intake of fruit and vegetables: the Hordaland Homocysteine Study. Am J Clin Nutr 81, 434-439.

41. Winkels RM, Brouwer IA, Clarke R, et al. (2008) Bread fortified with folic acid and vitamin B-12 improves the folate and vitamin B-12 status of healthy older people: a randomized controlled trial. Am J Clin Nutr 88, 348-355.

42. Ganji V \& Kafai MR (2004) Frequent consumption of milk, yogurt, cold breakfast cereals, peppers, and cruciferous vegetables and intakes of dietary folate and riboflavin but not vitamins B-12 and B-6 are inversely associated with serum total homocysteine concentrations in the US population. Am J Clin Nutr 80, 1500-1507.

43. Tamura T, Shin YS, Buehring KU, et al. (1976) The availability of folates in man: effect of orange juice supplement on intestinal conjugase. Br J Haematol 32, 123-133.

44. Tucker KL, Selhub J, Wilson PWF, et al. (1996) Dietary intake pattern relates to plasma folate and homocysteine concentrations in the Framingham Heart Study. J Nutr 126, 3025-3031.

45. Stea TH, Johansson M, Jagerstad M, et al. (2007) Retention of folates in cooked, stored and reheated peas, broccoli and potatoes for use in modern large-scale service systems. Food Chem 101, 1095-1107. 